\title{
HEATING SIGNATURES IN THE DISK COUNTERPARTS OF SOLAR SPICULES IN INTERFACE REGION IMAGING SPECTROGRAPH OBSERVATIONS
}

\author{
L. Rouppe van der Voort ${ }^{1}$, B. De Pontieu ${ }^{1,2}$, T. M. D. Pereira ${ }^{1}$, M. Carlsson ${ }^{1}$, and V. Hansteen ${ }^{1}$ \\ ${ }^{1}$ Institute of Theoretical Astrophysics, University of Oslo, P.O. Box 1029 Blindern, N-0315 Oslo, Norway \\ ${ }^{2}$ Lockheed Martin Solar \& Astrophysics Lab, Org. A021S, Bldg. 252, 3251 Hanover Street Palo Alto, CA 94304, USA \\ Received 2014 November 6; accepted 2014 December 15; published 2015 January 9
}

\begin{abstract}
We use coordinated observations with the Interface Region Imaging Spectrograph (IRIS) and the Swedish $1 \mathrm{~m}$ Solar Telescope to identify the disk counterpart of type II spicules in upper-chromospheric and transition region (TR) diagnostics. These disk counterparts were earlier identified through short-lived asymmetries in chromospheric spectral lines: rapid blue- or red-shifted excursions (RBEs or RREs). We find clear signatures of RBEs and RREs in $\mathrm{Mg}$ II $\mathrm{h} \& \mathrm{k}$, often with excursions of the central h3 and $\mathrm{k} 3$ absorption features in concert with asymmetries in co-temporal and co-spatial $\mathrm{H} \alpha$ spectral profiles. We find spectral signatures for RBEs and RREs in C II 1335 and $1336 \AA$ and Si IV 1394 and $1403 \AA$ spectral lines and interpret this as a sign that type II spicules are heated to at least TR temperatures, supporting other recent work. These C II and Si IV spectral signals are weaker for a smaller network region than for more extended network regions in our data. A number of bright features around extended network regions observed in IRIS slit-jaw imagery SJI 1330 and 1400, recently identified as network jets, can be clearly connected to H $\alpha$ RBEs and/or RREs in our coordinated data. We speculate that at least part of the diffuse halo around network regions in the IRIS SJI 1330 and 1400 images can be attributed to type II spicules with insufficient opacity in the C II and Si IV lines to stand out as single features in these passbands.
\end{abstract}

Key words: Sun: chromosphere - Sun: magnetic fields - Sun: transition region - Sun: UV radiation

Supporting material: animations

\section{INTRODUCTION}

Spicules are highly dynamic, linear features that can be observed as jet-like extrusions that emanate from the solar limb (for a recent review, see Tsiropoula et al. 2012). Two classes of spicules have been identified of which the second class is the most energetic displaying shortest lifetimes and most vigorous dynamical evolution (De Pontieu et al. 2007; Pereira et al. 2012). The complex dynamics of these type II spicules has been identified as the interplay between three kinds of motion: up flow, transversal, and torsional motions (De Pontieu et al. 2012). A recent study combing observations from Hinode and the Interface Region Imaging Spectrograph (IRIS) showed that type II spicules are heated to transition region (TR) temperatures (Pereira et al. 2014). This strengthens earlier observational indications that type II spicules are heated to coronal temperatures (De Pontieu et al. 2011).

The true impact of spicules on the corona in terms of provision of mass and energy is still unclear and a topic of debate. A prerequisite to realistic modeling of spicules is detailed knowledge of their physical properties. A full observational characterization is, however, hampered by lineof-sight confusion due to superposition effects at the limb. This problem is relieved on the solar disk where single spicules can be identified unambiguously. The disk counterparts of type II spicules have been identified through short-lived asymmetries of chromospheric spectral lines and are referred to as rapid blue- or red-shifted excursions (RBEs or RREs; Langangen et al. 2008; Rouppe van der Voort et al. 2009; Sekse et al. 2012, 2013a, 2013b; Yurchyshyn et al. 2013).

In this study we use coordinated observations from IRIS and the Swedish $1 \mathrm{~m}$ Solar Telescope (SST). RBEs and RREs are identified in the SST data and we search for corresponding signal in various IRIS diagnostics.

\section{OBSERVATIONS AND DATA PROCESSING}

We study quiet-Sun and coronal hole observations from a coordinated IRIS/SST observing campaign during the period 2013 August 25-October 3. IRIS observes spectra and slit-jaw images in a number of spectral windows in the near- and far-UV (for details, see De Pontieu et al. 2014b). Here we concentrate on spectral diagnostics of the transition region: Si IV 1394 and $1403 \AA$, the upper chromosphere/transition region: $C_{\text {II }} 1335$ and $1336 \AA$, and the chromosphere: $\mathrm{Mg}$ II h \& k (2803 and $2796 \AA$ A). Spectra were acquired with the IRIS slit at a fixed solar position (following solar rotation, a so-called "sit-andstare" program), or with narrow spatial rasters; Table 1 provides details for the different data sets. Besides the spectra, we analyze IRIS slit-jaw images (commonly referred to as SJI): SJI 1400 (dominated by the Si IV lines), SJI 1330 (dominated by the C II lines), and SJI 2796 (Mg II k core and inner wings). Exposure times for both spectra and slit-jaw images were $4 \mathrm{~s}$, and the pixel size 0 '. 166 . The IRIS observations were processed to level3 data (for more details, see De Pontieu et al. 2014b).

With the Crisp Imaging Spectropolarimeter (CRISP; Scharmer et al. 2008) at the SST (Scharmer et al. 2003), we acquired spectra in $\mathrm{H} \alpha$ and $\mathrm{Ca}$ II 8542, and Stokes V maps in Fe I 6302 at $-48 \mathrm{~m} \AA$ at a temporal cadence of about $11 \mathrm{~s} . \mathrm{H} \alpha$ was symmetrically sampled at 15 line positions, with $200 \mathrm{~m} \AA$ steps, Ca II 8542 at 25 line positions, with $100 \mathrm{~m} \AA$ steps. High spatial resolution was achieved with the aid of the adaptive optics system (recently upgraded with an 85 electrode deformable mirror), and with image restoration using the Multi-Object MultiFrame Blind Deconvolution (van Noort et al. 2005) method. The CRISP data reduction pipeline (de la Cruz Rodríguez et al. 2014) includes different methods described in detail by de la Cruz Rodríguez et al. (2013), Henriques (2012), and van Noort \& Rouppe van der Voort (2008). After data reduction, the effective 
Table 1

Overview of the IRIS Data Sets Analyzed in this Study

\begin{tabular}{|c|c|c|c|c|c|c|c|}
\hline \multirow[t]{2}{*}{ Date } & \multirow[t]{2}{*}{ Time (UT) } & \multirow[t]{2}{*}{ Type $^{\mathrm{a}}$} & \multirow[t]{2}{*}{$\mathrm{FOV}^{\mathrm{b}}$} & \multirow[t]{2}{*}{ Pointing } & \multicolumn{3}{|c|}{ Overlap with $\mathrm{SST}^{\mathrm{c}}$} \\
\hline & & & & & Time & SJI $\left[\operatorname{arcsec}^{2}\right]^{\mathrm{d}}$ & Slit \\
\hline 2013 Sep 13 & $08: 17-14: 54$ & Four-step dense raster & $1^{\prime \prime} \times 50^{\prime \prime}$ & Quiet Sun disk center & $02: 13$ & $2366(95 \%)$ & $43^{\prime \prime}$ \\
\hline 2013 Sep 18 & $08: 00-11: 30$ & Two-step sparse raster & $1^{\prime \prime} \times 50^{\prime \prime}$ & Quiet Sun $\left(37^{\prime \prime}, 58^{\prime \prime}\right)$ & $00: 11$ & $2204(88 \%)$ & $42^{\prime \prime}$ \\
\hline 2013 Sep 22 & $07: 34-11: 04$ & Medium sit-and-stare & $0.33 \times 61^{\prime \prime}$ & Coronal Hole $\left(538^{\prime \prime}, 283^{\prime \prime}\right)$ & 02:03 & $1372(38 \%)$ & $26^{\prime \prime}$ \\
\hline 2013 Sep 23 & 07:09-12:05 & Medium sit-and-stare & $0^{\prime \prime} 33 \times 61^{\prime \prime}$ & Quiet Sun disk center & $00: 46$ & $1323(37 \%)$ & $15^{\prime \prime}$ \\
\hline
\end{tabular}

Notes.

a A dense raster has 0.'33 slit steps, a sparse raster has $1^{\prime \prime}$ steps. A sit-and-stare program keeps the slit at a fixed position.

b Area covered by the spectrograph slit.

$\mathrm{c}$ The last three columns provide information on the overlap of the SST and IRIS data.

${ }^{\mathrm{d}}$ The overlap of SST was measured against SJI 2796 (percentage of SJI pixels covered in the SST FOV).
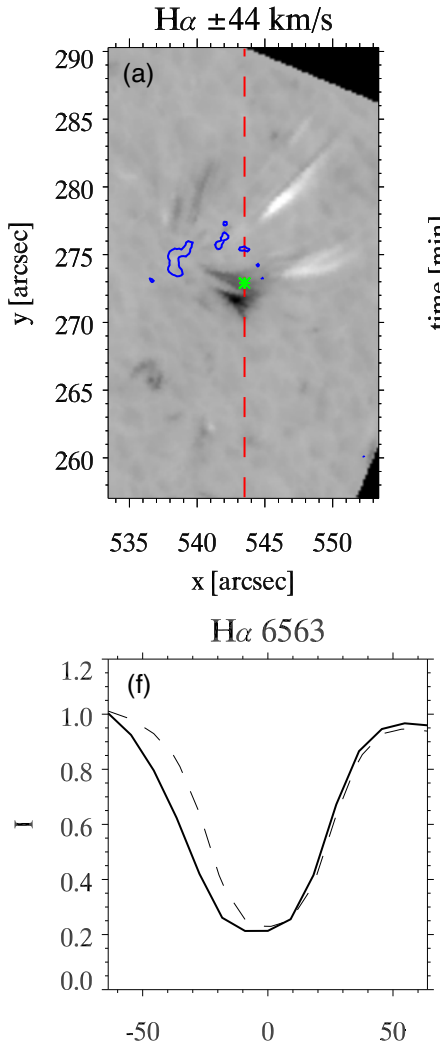

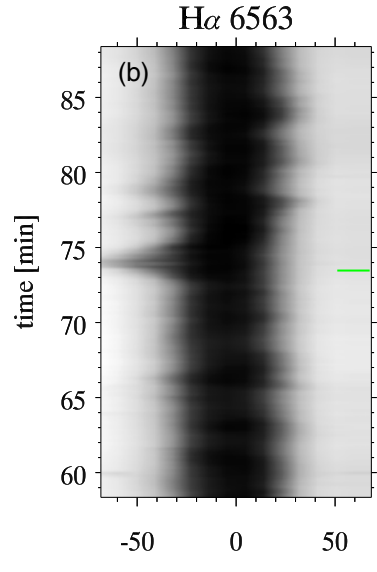

Mg II k 2796

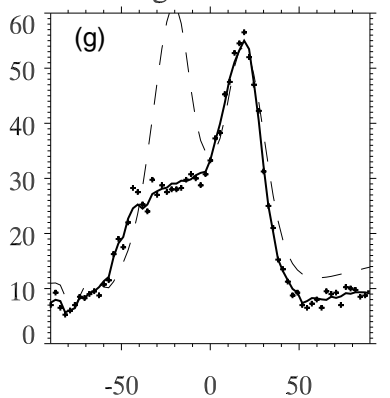

Mg II k 2796

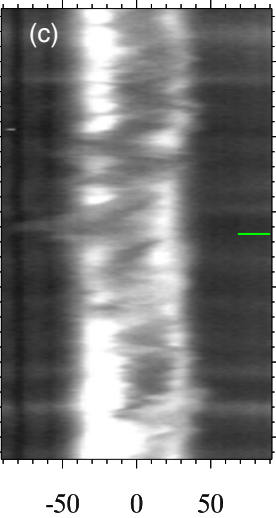

C II 1336

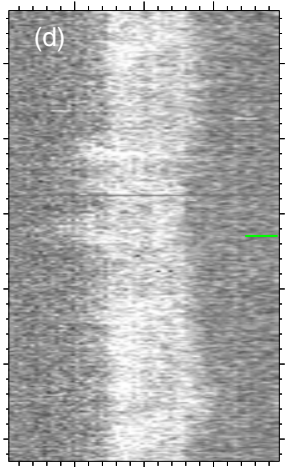

$\begin{array}{rrr}-50 & 0 & 50\end{array}$
Si IV 1394

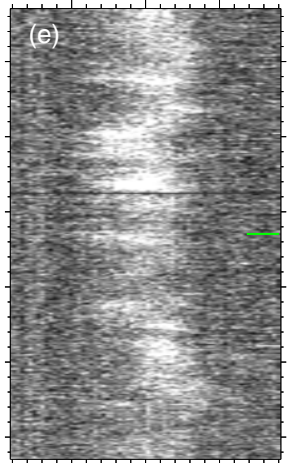

$\begin{array}{lll}-50 & 0 & 50\end{array}$ $\Delta \lambda[\mathrm{km} / \mathrm{s}]$

\section{II 1336}

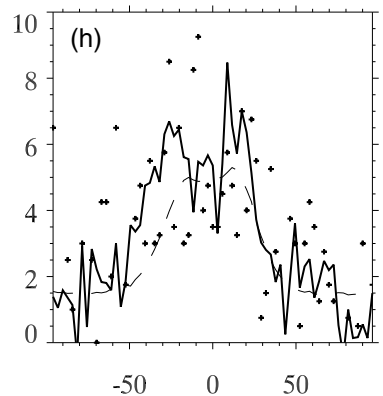

Si IV 1394

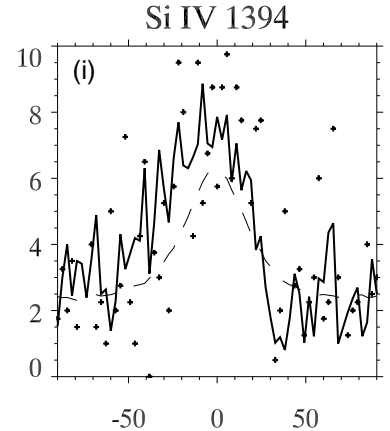

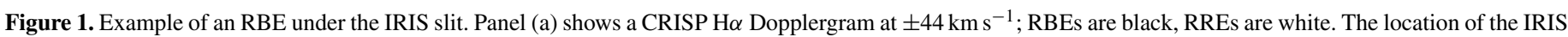

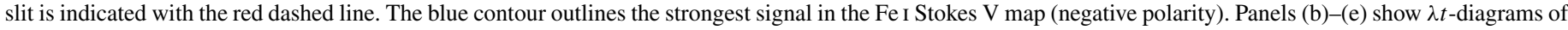

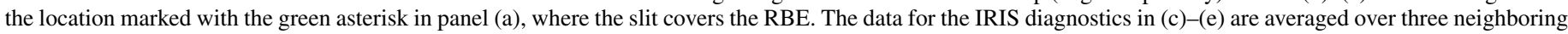

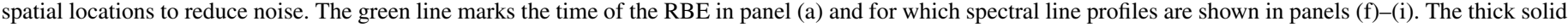

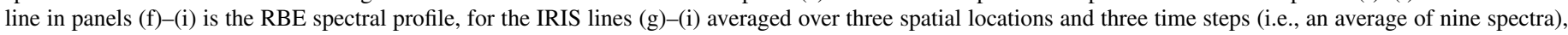

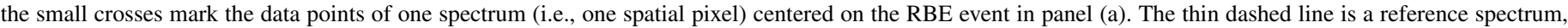
spatially and temporally averaged over slit positions far away from the network region.

(An animation of this figure is available.)

field of view (FOV) of the CRISP observations is approximately $55^{\prime \prime} \times 55^{\prime \prime}$, with a pixel scale of 0 '.058.

Alignment of the SST and IRIS data was done by scaling down the CRISP data to IRIS pixel scale and by cross-correlation of Ca II 8542 far wing images with IRIS SJI 2796 images. This alignment procedure proved to be accurate down to the level of the IRIS pixel scale. Details of the overlap of SST and IRIS data are given in the last three columns of Table 1. For exploration of the aligned SST and IRIS data, we used CRISPEX (Vissers \& Rouppe van der Voort 2012), initially developed for CRISP data and now expanded to browse IRIS level3 data. CRISPEX is part of SolarSoft.

\section{RESULTS}

RBEs and RREs are most easily recognized in $\mathrm{H} \alpha$ Doppler maps (blue - red far wing subtraction images) and are most frequently found around network regions. In the 2013 September 22 data set, the IRIS slit was positioned in the vicinity of a small network patch and we observe a large number ( $>150$ in $2 \mathrm{hr}$ ) of RBEs/RREs that are covered by the IRIS slit. This is illustrated in $\mathrm{H} \alpha \pm 44 \mathrm{~km} \mathrm{~s}^{-1}$ Doppler maps in panels (a) of Figures 1 and 2 and the accompanying animation: RBEs (dark streaks) and RREs (white) appear to originate radially from the region around $(x, y) \approx\left(538^{\prime \prime}, 275^{\prime \prime}\right)$ and cross the IRIS slit (red dashed 

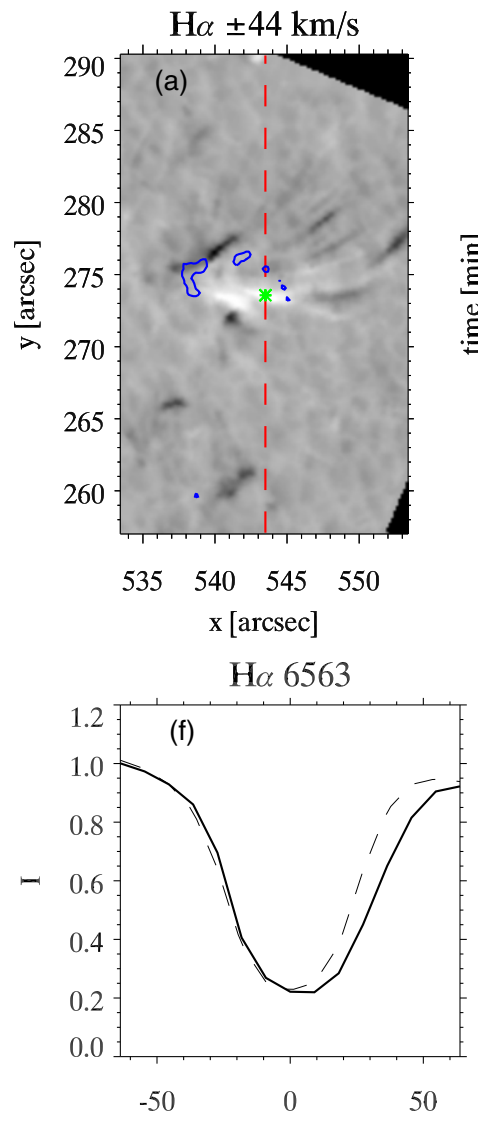

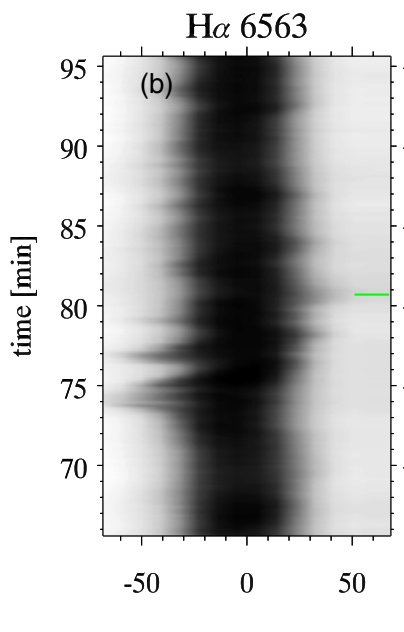

Mg II k 2796

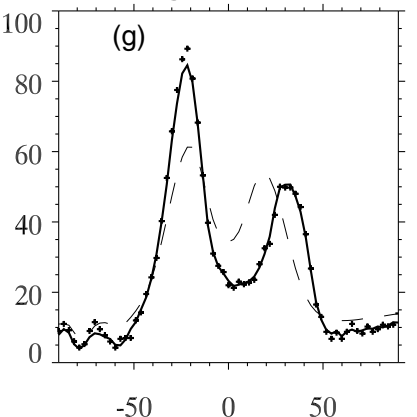

Mg II k 2796

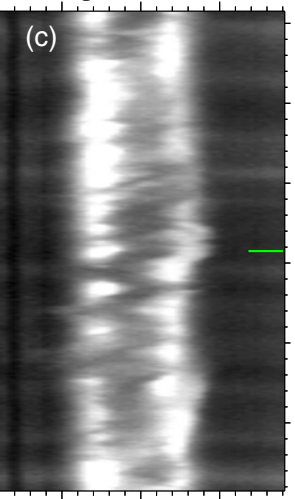

$\begin{array}{lll}-50 & 0 & 50\end{array}$

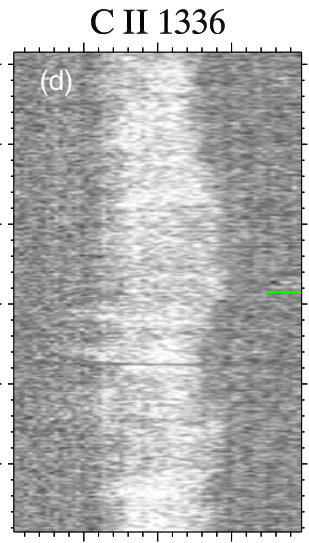

$\begin{array}{lll}-50 & 0 & 50\end{array}$

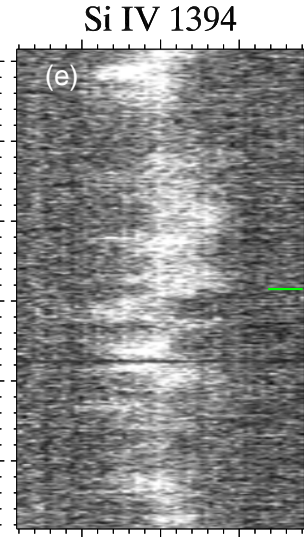

$\begin{array}{rrr}-50 & 0 & 50\end{array}$ $\Delta \lambda[\mathrm{km} / \mathrm{s}]$

$$
\text { C II } 1336
$$

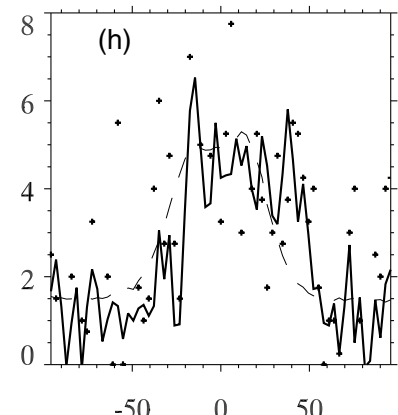

$\begin{array}{lll}-50 & 0 & 50\end{array}$

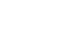

$10+$

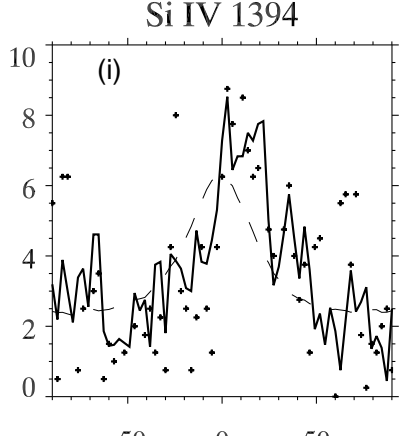

$\Delta \lambda[\mathrm{km} / \mathrm{s}]$

Figure 2. Example of an RRE under the IRIS slit. The panel layout is the same as in Figure 1.

line). The spectral evolution ( $\lambda t$-diagrams) of one location at the slit is shown in panels (b)-(e). The H $\alpha$ t-diagram ((b)) shows the occurrence of a large number of RBEs and RREs at this location; short-lived asymmetry excursions of the line, most prominently the RBE around $t=74$ minutes, the time of the Dopplergram in Figure 1(a), which shows the RBE as a dark streak crossed by the IRIS slit at the green asterisk. With the blue- and red-shifted excursions in the $\mathrm{H} \alpha \lambda t$-diagram at hand, the RBEs and RREs can be readily identified as similar excursions of the central absorption feature $\mathrm{k} 3$ in the $\mathrm{Mg}$ II $\mathrm{k}$ diagram (c). The shift of $\mathrm{k} 3$ leads to the enhancement of the wing so that the RBE/RREs appear as emission features far out in the wings. The line profile in Figure 1(g) shows the RBE as a blue-shift of $\mathrm{Mg}$ II $\mathrm{k} 3$ and absence of a prominent $\mathrm{k} 2 \mathrm{v}$ peak as compared to the average $\mathrm{Mg}$ II $\mathrm{k}$ profile (constructed from temporal and spatial averaging over slit positions far away from the network region). For the RRE example in Figure 2, the k2r peak is weakened but not absent, and shifted with more than $10 \mathrm{~km} \mathrm{~s}^{-1}$ as compared to the average profile. Even more than the RBE example in Figure 1, this RRE appears as an emission feature extending out to $\sim+40 \mathrm{~km} \mathrm{~s}^{-1}$ in the $\lambda t$-diagram (c).

The far-UV spectra for C II $1336 \AA$ and Si IV $1394 \AA$ are very noisy at this exposure time. However, comparing panels (d) and (e) with (b) and (c), these lines show similar temporal behavior with blue- and red-ward asymmetry excursions in concert with their pure chromospheric counterparts $\mathrm{H} \alpha$ and $\mathrm{Mg}$ II k. After a nine-fold averaging (three slit positions over three time steps), the line profiles (solid lines in panels (h) and (i)) show asymmetry (and shift) toward the blue for the RBE and toward the red for the RRE. Single Gaussian fits to the UV spectral profiles indicate shifts in the range $11-14 \mathrm{~km} \mathrm{~s}^{-1}$ for both the RBE and RRE in Figures 1 and 2.

As the signals in these far-UV IRIS diagnostics are low, the associated slit-jaw images SJI 1330 and SJI 1400 show no sign of anything related to the RBEs and RREs that are so prominent in the CRISP data in this region. This network region can be considered to be weak in a sense that it is only a collection of a small number of photospheric magnetic bright points. However, in other more extended network regions with larger numbers of bright points, the slit jaw images show a pattern of a diffuse halo around the network patch, frequently interspersed with dynamic bright streaks that often appear to move away from the network region. These streaks are recently described as network jets by Tian et al. (2014). Many of these network jets can be directly related to RBEs and RREs in the CRISP Doppler animations: they can often be associated with an RBE or RRE in close vicinity with similar orientation and temporal evolution (life time and direction of propagation).

Figure 3 shows two examples of far-UV slit jaw jets in the 2013 September 13 quiet-Sun data set. Panel (d) shows a SJI 1330 jet, panel (a) shows the associated $\mathrm{H} \alpha \mathrm{RBE}$ in the cotemporal CRISP Doppler map, and panels (b) and (e) show the co-temporal spectrograms ( $\lambda y$-diagrams). In contrast with the spectra in Figures 1 and 2, the Si IV $1394 \AA$ and C II $1336 \AA$ spectra show strong signal with clearly shifted and blue-ward asymmetric line profiles. Gaussian fits to these line profiles indicate blue shifts of $-16 \mathrm{~km} \mathrm{~s}^{-1}$ for $\mathrm{Si} I \mathrm{IV}$ and $-12 \mathrm{~km} \mathrm{~s}^{-1}$ for $\mathrm{C}_{\text {II }}$ with respect to the average line profiles. We note the asymmetry of the profiles toward higher Doppler shift that is not captured by a single Gaussian profile. 

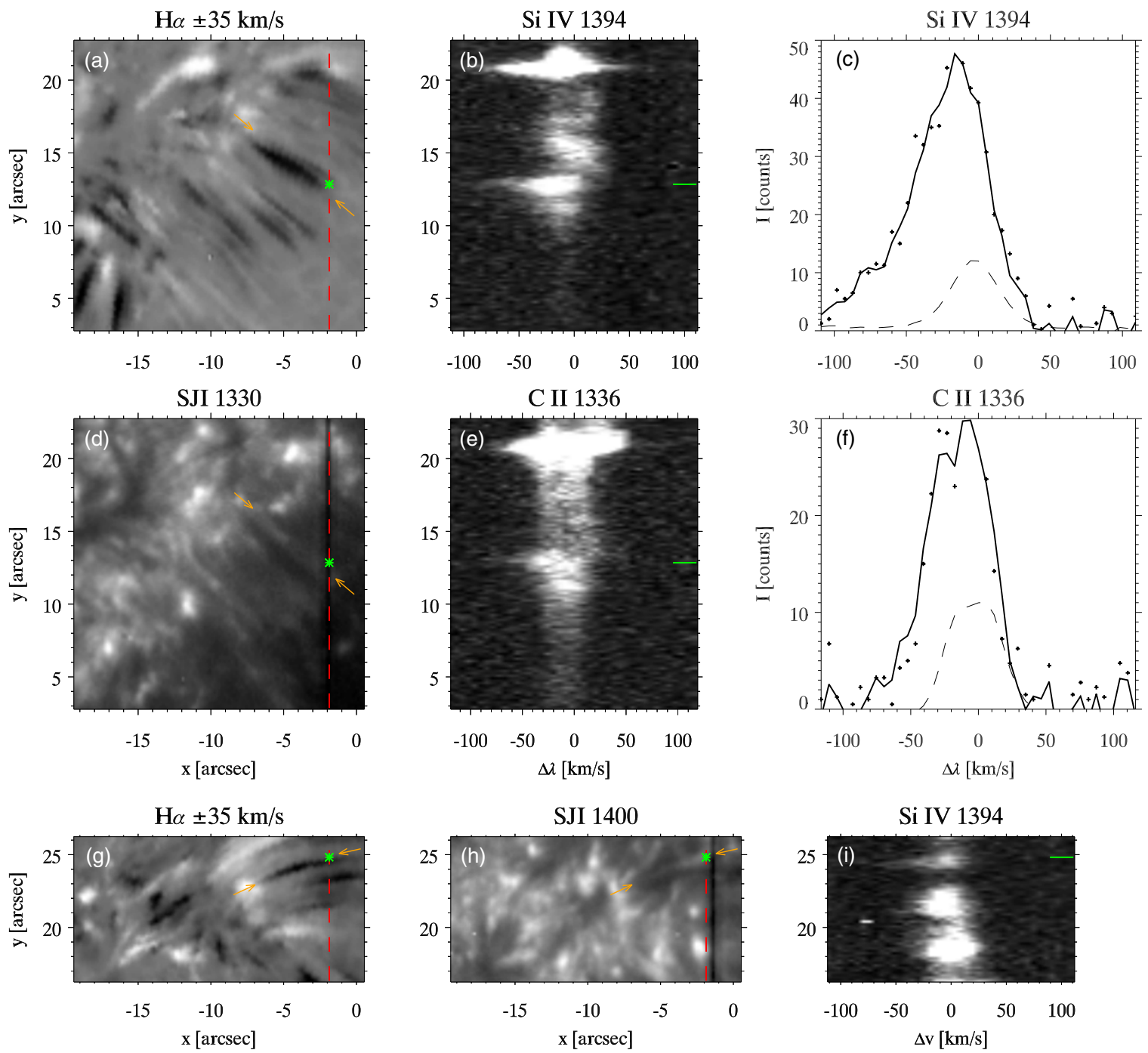

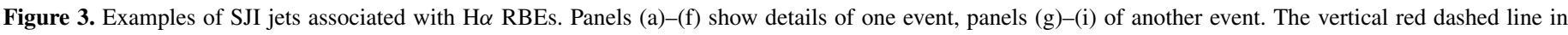

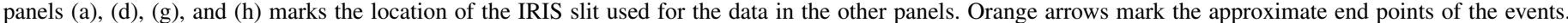

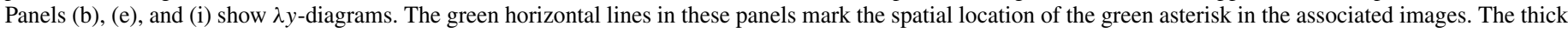

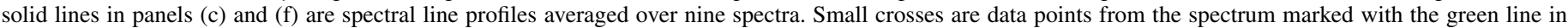
panels (b) and (e). The thin dashed lines are reference spectra.

The bottom three panels in Figure 3 show another example: a bright SJI 1400 jet (h), associated with an $\mathrm{H} \alpha \mathrm{RBE}$ in (g) and a strong asymmetric profile in the Si Iv $1394 \AA$ A spectrogram.

Figure 4 shows four more examples of jets in SJI 1330 or SJI 1400 associated with RBEs or RREs in H $\alpha$ Doppler maps. The features of interest are marked with arrows in the different panels, but the association between IRIS SJI jets and CRISP RBE/RREs is most vivid in the accompanying animations available as supporting material.

\section{DISCUSSION}

We use coordinated observations from the SST and IRIS to identify the disk counterparts of type II spicules in various IRIS chromospheric and TR diagnostics. One of the data sets is a more than $2 \mathrm{hr}$ time series with the IRIS spectrograph slit positioned in the proximity $\left(\sim 5^{\prime \prime}\right)$ of a small network region in a coronal hole. A large number of $\mathrm{H} \alpha$ RBEs and RREs ( $>150)$ were found to be covered by the IRIS slit. For these events, we find clear correspondence between the temporal behavior of the $\mathrm{H} \alpha$ line and the $\mathrm{Mg}$ II $\mathrm{h} \& \mathrm{k}$ lines: for $\mathrm{H} \alpha$ RBEs we find corresponding $\mathrm{Mg}$ blue-shifted spectral features and for RREs we find corresponding red-shifted features. We observe that the $\mathrm{k} 3$ and $\mathrm{h} 3$ central absorption dips show similar Doppler excursions as the asymmetries in the $\mathrm{H} \alpha$ line. This often leads to a strong reduction (sometimes almost complete removal) of one of the $\mathrm{k} 2$ and $\mathrm{h} 2$ reversal peaks accompanied with a Doppler shift of this peak. The shift results in enhanced emission in the wing so that RBE/RREs can be observed as bright features in the $\mathrm{Mg}$ II $\mathrm{h} \& \mathrm{k}$ wings. This is different from $\mathrm{H} \alpha$ and $\mathrm{Ca}$ II $8542 \AA$ where RBE/RREs are pure absorption features and therefore appear dark in wing images.

With $4 \mathrm{~s}$ exposure times, the $\mathrm{C}_{\mathrm{II}}$ and Si IV spectra in this coronal hole region are to a large extent dominated by noise. However, after averaging of spectra (both spatially and temporally), we are able to identify spectral signatures for these lines that can be associated with $\mathrm{H} \alpha$ and Mg RBEs and RREs. We often see (weak) enhanced emission with corresponding Doppler shift for both the $\mathrm{C}$ II and Si IV lines. In a different data set, with the IRIS slit positioned in the proximity of a more extended magnetic network region in a quiet-Sun region, we observe for some $\mathrm{H} \alpha$ RBEs and RREs much stronger response in these 

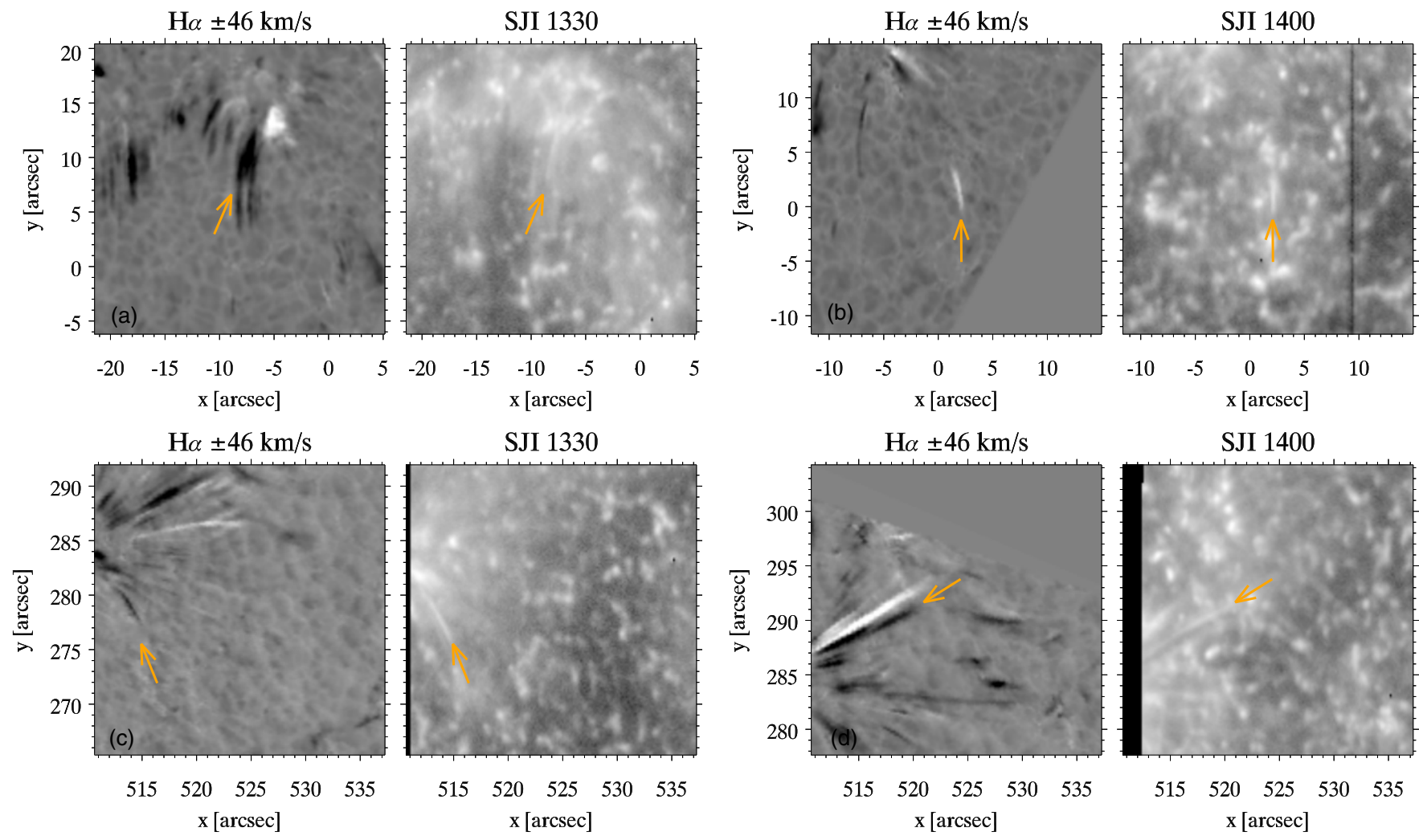

Figure 4. Four examples of SJI jets associated with $\mathrm{H} \alpha$ RBEs or RREs. Orange arrows guide the eye to identify the events in both images for each pair. (Animations of this figure are available.)

upper-chromospheric and TR diagnostics. For these events, no spectral averaging is needed and the presence of $\mathrm{C}_{\text {II }}$ and Si IV signal corresponding to $\mathrm{H} \alpha$ RBEs or RREs is unquestionable.

The RBEs and RREs that have strong C II and Si IV response can be clearly identified as bright streaks in the SJI 1330 and 1400 slit-jaw images. These kind of bright streaks can be found around the stronger network regions anywhere on the solar disk in the IRIS SJI 1330 and 1400 data, Tian et al. (2014) describe these features as network jets. When we have coordinated SST $\mathrm{H} \alpha$ observations, we can often identify corresponding RBEs and RREs that display striking similarity in morphology and dynamical evolution. This suggests that we observe the same feature in different diagnostics. Here we present four examples of $\mathrm{H} \alpha \mathrm{RBE} / \mathrm{RREs}$ that can be associated with SJI 1330 or 1400 jets. De Pontieu et al. (2014a) present three other events from the same data sets for which SJI 1330 and 1400 jets are associated with RBE/RREs that display clear twist in their dynamical evolution. The relatively broad bandpasses for the SJI 1330 and 1400 filters (55 A for both) cover a large continuum window. The strong line emission we observe for jets that are covered by the IRIS slit indicates that the network jets in the slit jaw data are dominated by line emission rather than continuum contribution.

We conclude that our observations show that type II spicules are heated to at least TR temperatures, interpreting the Si IV emission as signs of $\sim 80 \mathrm{kK}$ plasma (under equilibrium conditions). This is in agreement with Pereira et al. (2014), who tied Hinode Ca II $\mathrm{H}$ type II spicules at the quiet-Sun limb to IRIS SJI 1400 spicules. Their study demonstrates that the Hinode $\mathrm{Ca} \mathrm{H}$ passband shows type II spicules only during their initial (cooler) phase and that the spicules continue to evolve in the hotter SJI 1400 passband. Furthermore, Pereira et al. (2014) identified RBE and RRE signatures in Mg II k spectroheliograms from dense IRIS rasters close to the limb. Many of these $\mathrm{Mg}$ RBEs and RREs could be seen to continue in Si IV spectroheliograms. Our study supports these results and further establish a close connection between RBE/RREs observed in $\mathrm{H} \alpha$ and $\mathrm{Mg}$ II h \& k.

We note the difference between the small network region close to the IRIS slit in the 2013 September 22 coronal hole data set and the more extended network regions in the same and other data sets. For the small network region, we only find weak $\mathrm{C}_{\text {II }}$ and Si IV signal and an absence of network jets in SJI 1330 and 1400. For the more extended network regions, we find network jets and associated features in the spectrograms. In the CRISP $\mathrm{H} \alpha$ and $\mathrm{Ca}$ II 8542 data, however, we cannot identify a clear difference in RBE/RRE activity between these network regions. Apparently, while these network regions seem indistinguishable in chromospheric diagnostics, for the smaller network region insufficient opacity is built up in C II and Si IV. Whether this is due to a different level of heating or mass loading can only be speculated on.

The network jets appear often to be embedded in diffuse halos that surround the larger network regions in SJI 1330 and 1400 data, while these halos appear to be absent in small network regions like in the 2013 September 22 data set. Apparently, the weak C II and Si IV emission that we effectively find for almost all RBEs and RREs is insufficient to give discernible signal in the slit-jaw data around small network regions. It might, however, be well possible that the diffuse halo around the larger network regions result from type II spicules with insufficient opacity to stand out as individual network jets in the slit jaw images.

We find a number of clear examples of SJI 1330 and 1400 network jets that are connected to $\mathrm{H} \alpha$ RBEs and/or RREs. However, we cannot firmly establish a chromospheric RBE/RRE 
connection for all network jets in our data. It is possible that this is due to a line-of-sight effect: some network jets might follow a dynamic trajectory that is unfavorable for identification in $\mathrm{H} \alpha$ wing Dopplergrams. Furthermore, it cannot be excluded that some network jets simply do not have a chromospheric component or are observed during a more energetic part of their evolution that makes it difficult to connect to a possible earlier chromospheric phase. Tian et al. (2014) connect network jets to the solar wind and measure apparent velocities that are often well above $100 \mathrm{~km} \mathrm{~s}^{-1}$. These apparent velocities are on the high end of what has been found earlier for RBE/RREs and type II spicules. The Doppler shifts we measure for C II and Si IV are in the low range of what was found for RBE/RREs (Sekse et al. 2013a). We note, however, that single Gaussian fits do not catch the full complexity of these profiles. For example, there appears to be an extra component at $\sim-75 \mathrm{~km} \mathrm{~s}^{-1}$ in the far blue wing of the Si IV profile in Figure 3(c). Further effort is required to explore how the asymmetry in the profiles can be characterized. This is crucial in order to determine to what extent the high apparent speeds in network jets are real mass flows.

In this Letter, we do not address the temporal evolution of the spicules in our coordinated data set. We note, however, the prominent appearance of swings between red and blue shifts in the $\mathrm{Mg}$ II $\lambda t$-diagrams. It seems that this temporal variation is much more clearly tractable than in the ground-based $\mathrm{H} \alpha$ and $\mathrm{Ca}$ II 8542 data. Potentially, this opens a new diagnostic window on the Alfvénic wave modes that govern the dynamics of spicules. However, disentangling the observed Doppler shifts in uniquely determined kink, torsional and up-flow components will remain to be a formidable challenge.

We thank J. De la Cruz-Rodríguez, T. Golding, C. Hoffman, S. Jafarzadeh, L. Kleint, A. Ortiz, E. Scullion, T. Tarbell, A. SainzDalda, and H. Skogsrud for assistance with SST observations and data processing. IRIS is a NASA Small Explorer mission developed and operated by LMSAL with mission operations executed at NASA ARC and major contributions to downlink communications funded by the NSC (Norway). The Swedish
$1 \mathrm{~m}$ Solar Telescope is operated on the island of La Palma by the Institute for Solar Physics (ISP) of Stockholm University in the Spanish Observatorio del Roque de los Muchachos of the Instituto de Astrofísica de Canarias. The authors gratefully acknowledge support from the International Space Science Institute (ISSI). This research was supported by the Research Council of Norway and by the European Research Council under the European Union's Seventh Framework Programme (FP7/2007-2013)/ERC grant agreement No. 291058. B.D.P. is supported by NASA contract NNG09FA40C (IRIS), and NASA grants NNX11AN98G and NNM12AB40P.

\section{REFERENCES}

de la Cruz Rodríguez, J., Löfdahl, M., Sütterlin, P., Hillberg, T., \& Rouppe van der Voort, L. 2014, A\&A, 573, A40

de la Cruz Rodríguez, J., Rouppe van der Voort, L., Socas-Navarro, H., \& van Noort, M. 2013, A\&A, 556, A115

De Pontieu, B., Carlsson, M., Rouppe van der Voort, L. H. M., et al. 2012, ApJL, 752, L12

De Pontieu, B., McIntosh, S., Hansteen, V. H., et al. 2007, PASJ, 59, 655

De Pontieu, B., McIntosh, S. W., Carlsson, M., et al. 2011, Sci, 331, 55

De Pontieu, B., Title, A., \& Carlsson, M. 2014a, Sci, 346, D315

De Pontieu, B., Title, A. M., Lemen, J. R., et al. 2014b, SoPh, 289, 2733

Henriques, V. M. J. 2012, A\&A, 548, A114

Langangen, Ø., De Pontieu, B., Carlsson, M., et al. 2008, ApJL, 679, L167

Pereira, T. M. D., De Pontieu, B., \& Carlsson, M. 2012, ApJ, 759, 18

Pereira, T. M. D., De Pontieu, B., Carlsson, M., et al. 2014, ApJL, 792, L15

Rouppe van der Voort, L., Leenaarts, J., de Pontieu, B., Carlsson, M., \& Vissers, G. 2009, ApJ, 705, 272

Scharmer, G. B., Bjelksjö, K., Korhonen, T. K., Lindberg, B., \& Petterson, B. 2003, Proc. SPIE, 4853, 341

Scharmer, G. B., Narayan, G., Hillberg, T., et al. 2008, ApJL, 689, L69

Sekse, D. H., Rouppe van der Voort, L., \& De Pontieu, B. 2012, ApJ, 752, 108

Sekse, D. H., Rouppe van der Voort, L., \& De Pontieu, B. 2013a, ApJ, 764, 164 Sekse, D. H., Rouppe van der Voort, L., De Pontieu, B., \& Scullion, E. 2013b, ApJ, 769, 44

Tian, H., DeLuca, E. E., Cranmer, S. R., et al. 2014, Sci, 346, 1255711

Tsiropoula, G., Tziotziou, K., Kontogiannis, I., et al. 2012, SSRv, 169, 181

van Noort, M., Rouppe van der Voort, L., \& Löfdahl, M. G. 2005, SoPh, 228,191

van Noort, M. J., \& Rouppe van der Voort, L. H. M. 2008, A\&A, 489, 429

Vissers, G., \& Rouppe van der Voort, L. 2012, ApJ, 750, 22

Yurchyshyn, V., Abramenko, V., \& Goode, P. 2013, ApJ, 767, 17 